\title{
A Trust Brokering System and Its Application to Resource Management in Public-Resource Grids
}

\author{
Farag Azzedin \\ Dept. of Computer Science \\ University of Manitoba \\ Winnipeg, MB R3T 2N2, Canada \\ fazzedin@cs.umanitoba.ca
}

\author{
Muthucumaru Maheswaran \\ School of Computer Science \\ McGill University \\ Montreal, QC H3A 2A7, Canada \\ maheswarecs.mcgill.ca
}

\begin{abstract}
This paper presents a trust brokering system that operates in a peer-to-peer manner. The network of trust brokers operate by providing peer reviews in the form of recommendations regarding potential resource targets. One of the distinguishing features of our work is that it separately models the accuracy and honesty concepts. By separately modeling these concepts, our model is able to significantly improve the performance. We apply the trust brokering system to a resource manager to illustrate its utility in a publicresource Grid environment. The simulations performed to evaluate the trust-aware resource management strategies indicate that high levels of "robustness" can be attained by considering trust while allocating the resources.
\end{abstract}

\section{Introduction}

The Grid computing system $[11,8]$ is a highly scalable network computing system that is emerging as a popular mechanism for harnessing resources that are distributed on a wide-area network. Conceptually, a Grid computing system allows resources from different administrative domains to participate in it and ensures the autonomy of the different sites (referred hereafter as domains). However, in current practice, Grid computing systems are built from resources that are contributed by institutions that agree to work together due to off-line trust relationships that exist or are forged among them. To scale a Grid beyond hundreds of nodes, it is necessary to accommodate "public" resources, where a priori trust relationships do not exist among the resources [7].

The term public-resource Grids refers to a class of Grids, where the resources do not have a priori trust relationships. A variety of different approaches can be used to construct Grid systems that fit into this class including: (a) combining Grids and P2P systems and (b) generalizing P2P systems with resource management systems. One of the most desir- able features of the public-resource Grids is that it opens up the membership of the Grid very much like P2P file sharing systems. This provides an opportunity for the Grid to scale in terms of participants and increases the eligible Grid participants. Currently, only best-effort application specific Grids such as SETI@home bring public resources under a single virtual entity. One way of increasing the applicability of public-resource Grids is to make them QoS aware.

To provide services with QoS, the resources should be managed. Because a public-resource Grid is made of resources with heterogeneous trust relationships [7, 6, 18], the resource manager needs to consider these relationships while managing the resources.

This paper contributes to this important problem in the following ways: (a) presents a trust brokering system that manages and models the trust relationships among the different parts of the network computing system, (b) devises new mechanisms for efficiently maintaining recommendation based systems, and (c) introduces a new methodology for incorporating trust into resource management systems that is based on risk minimization.

Our trust broker system is based on a trust model that separates the concepts of accuracy and honesty. The concept of accuracy enables peer review-based mechanisms to function with imprecise trust metrics, where different peers can evaluate the same situation differently. By introducing the concept of honesty, we handle the situation where peers intentionally lie about others for their own benefit. Using simulations, we demonstrate that these two conditions can be handled by our mechanisms and the importance of properly handling these conditions.

Section 2 defines the notions of trust and reputation and outlines mechanisms for computing them. In Section 3, we describe the architecture of the trust brokering system. Section 4 discusses the results from a simulation study performed to evaluate the proposed trust model. Section 5 presents a case study designed to investigate the utility of 
the proposed trust model. Related work is briefly discussed in Section 6.

\section{Trust Model}

\subsection{Fundamental Trust Concepts}

In this paper, behavior trust is quantified by the dynamic parameter trust level (TL) that ranges from very untrustworthy $(\mathrm{TL}=1)$ to very trustworthy $(\mathrm{TL}=5)$. The $\mathrm{TL}$ is computed based on past experiences for a specific context. Ideally, reputation of an entity $y$ is the behavior trust value reached by global consensus. In practice, it is estimated by polling sufficiently large number of recommenders regarding the behavior trust of entity $y$. Because recommenders can be dishonest and distort the reputation estimates, we introduce an honesty concept that tracks the truthfulness of a recommender. A recommender is considered to be truthful if it says what it actually knows. Accuracy is another important concept that tracks how correctly a recommender estimates the underlying trust values.

\subsection{Assumptions and Trust Model Elements}

Our trust model assumes that each entity $x$ maintains a set of recommenders $(R)$ and a set of trusted allies $(T)$. Entity $x$ completely trusts its trusted allies that are chosen based on off-line trust relationships. Trusted allies are used by an entity to determine the honesty of its recommenders. In general, trusted allies of an entity $x$ do not have sufficient knowledge to provide recommendations themselves. The recommenders of $x$ are maintained in a recommender trust table (RTT), where a two-tuple (honesty, accuracy) is associated with each entry. The initial membership of RTT is randomly chosen and it evolves as described below. Similarly, $x$ maintains another table called direct trust table (DTT) for tracking transactions that $x$ had with other entities. In addition to the above, we make the following assumptions as well: (a) behavior trust is a slowly varying parameter, (b) transactions between entities are secure and the source and destination are properly authenticated, and (c) trustworthiness and honesty are independent notions.

\subsection{Computing Honesty and Accuracy}

To determine the honesty of recommender $z$, entity $x$ instructs the entities in its $T$ to request recommendations from $z$ regarding entity $y$ for a specific context. These requests are launched such that they arrive at $z$ as closely spaced in time as possible. Because behavior trust is a slow varying parameter, if $z$ is honest, it cannot give away largely different answers. Let the honesty of recommender $z$ as observed by entity $x$ be denoted as $H(x, z)$ and let $R E_{k}(z, y, t, c)$ denote the recommendation for entity $y$ given by $z$ to entity $k$ for context $c$ and time $t$, where $k \in T$. Let $T L_{\text {min }}=\min _{k \in T}\left\{R E_{k}(z, y, t, c)\right\}$ and $T L_{\max }=\max _{k \in T}\left\{R E_{k}(z, y, t, c)\right\}$. Let $\Delta_{R E}$ denote the difference and be given by $\Delta_{R E}=T L_{\max }-T L_{\min }$. The value of $\Delta_{R E}$ will be less than a small value $\epsilon_{R E}$ if recommender $z$ is honest. Consequently, $H(x, z)$ is computed as follows:

$$
H(x, z)= \begin{cases}0 & \text { if } \Delta_{R E}>\epsilon_{R E} \\ 1 & \text { otherwise }\end{cases}
$$

Let the accuracy of recommender $z$ as observed by entity $x$ for a specific context $c$ at a given time $t$ be denoted as $A(x, z, t, c)$, where $0 \leq A(x, z, t, c) \leq 1$. Let $T T L_{x}(y, t, c)$ denote the true trust level (TTL) of $y$ obtained by $x$ as a result of monitoring its transaction with $y$ for context $c$ at time $t$. Let $\Psi_{R E}=R E_{x}(z, y, t, c)-$ $T T L_{x}(y, t, c)$. The value of $\left|\Psi_{R E}\right|$ is an integer value ranging from 0 to 4 because $R E_{x}(z, y, t, c)$ and $T T L_{x}(y, t, c)$ are in [1..5]. Then, $A(x, z, t, c)$ can be computed as:

$$
A(x, z, t, c)=-\frac{1}{4}\left|\Psi_{R E}\right|+1
$$

Monitoring each transaction is an onerous task. Therefore, Equation (1) will be used to update accuracy every $n$th transaction and a weighted averaging process as shown in Section 4.3.1 will be used to maintain this parameter in between the updates. Before $x$ can use $R E_{x}(z, y, t, c)$ to calculate the reputation of $y, R E_{x}(z, y, t, c)$ must be adjusted to reflect recommender $z$ 's accuracy. Therefore, a shift function $(S)$ that uses the accuracy $A(x, z, t, c)$ to correct $R E_{x}(z, y, t, c)$ is formulated as follows:

$$
\begin{array}{r}
S\left(A(x, z, t, c), R E_{x}(z, y, t, c)\right) \\
= \begin{cases}R E_{x}(z, y, t, c)+4(1-A(x, z, t, c)) & \text { if } \Psi_{R E}^{*}<0 \\
R E_{y}(z, y, t, c)-4(1-A(x, z, t, c)) & \text { if } \Psi_{R E}^{*} \geq 0\end{cases}
\end{array}
$$

Because monitoring is done every $n$th transaction, $\Psi_{R E}^{*}$ is equal to the $\Psi_{R E}$ that was obtained at the last monitoring event.

\subsection{Computing Trust and Reputation}

The trust level that quantifies behavior trust between two entities is assumed to be made up of direct trust and reputation. Let the behavior trust for a given context $c$ and time $t$ between two entities $x$ and $y$ be $\Gamma(x, y, t, c)$, direct trust between the entities for the same context and time be $\Theta(x, y, t, c)$, and the reputation of $y$ for the same context and time be $\Omega(y, t, c)$. Let the weights given to direct and reputation trusts be $\alpha$ and $\beta$, respectively such that $\alpha+\beta=1$ and $\alpha, \beta \geq 0$. If the trustworthiness of $y$ (as far as $x$ is concerned) is based more on direct relationship with $x$ than the reputation of $y$ then $\alpha$ should be larger than $\beta$.

The reputation of $y$ is computed as the average of the product of the trust level in the DTT shifted by the shift function $S$, for all recommenders $z \in R \neq x$. In practice, DTT will be used to give recommendations and obtain direct trust levels.

$$
\begin{aligned}
\Gamma(x, y, t, c) & =\alpha \Theta(x, y, t, c)+\beta \Omega(y, t, c) \\
\Theta(x, y, t, c) & =\operatorname{DTT}(x, y, t, c)
\end{aligned}
$$


One way of estimating the reputation $\Omega(y, t, c)$ is to use recommendations. Let $\Omega_{x}(y, t, c)$ be the estimate of $\Omega(y, t, c)$ computed by $x$ based on the recommendations it received from its recommenders. In general, this estimate will not be the same as the actual value $\Omega(y, t, c)$. However, as a simplification measure, we assume the estimate to be sufficiently accurate.

$$
\Omega_{x}(y, t, c)=\frac{\sum_{z \in R} S\left(A(x, z, t, c), R E_{x}(z, y, t, c)\right)}{|R|},
$$

where $z \neq y$

\section{The Trust Brokering System 3.1. Trust Brokering Model}

The core of the trust brokering model is a peer-to-peer network of trust brokers (hereafter referred to as brokers), where each broker represents a portion of the network computing system called the domain. A broker is responsible for managing the trust of the resources and clients that are within its domain. Hence, the trust of a resource is managed by a single broker whereas a broker manages the trust of all the resources within its domain. The resources within a single domain are grouped into different classes by their expected reputation by the broker. A broker's reputation will depend on how accurate and honest it is in representing the reputations of its resources. For instance, when a resource misbehaves despite it being presented as a highly reputed resource by the broker, the reputation of the broker will be reduced. Conversely, if a broker conservatively estimates the reputation of its resources, then the resources within its purview can be unnecessarily shunned by other resources. Therefore, a broker is compelled to place a resource in the most appropriate class by weighing these conflicting requirements. When a resource joins a domain, it negotiates with the broker the trust level (reputation) that will be placed on it. The resource could use recommendations from prior broker associations to lay its claim for higher trust levels.

Often a broker may need to know about resources that are under the purview of brokers with whom it does not have any relationships. In this case, a broker will request recommendations regarding the target broker from its peering brokers. The predicted reputation of the target resource will depend on the reputation of the broker that manages it and the reputation bestowed upon the it by the broker. The post mortem analysis of the transactions will determine the validity of the predictions. The reputations and other trust levels of the brokers are adjusted based on the match between the predicted values and post-mortem detected values.

\subsection{Trust Representation and Usage}

The trust that exists among the brokers is represented by a DTT, where a specific row of the DTT shows how a particular source broker (represented as $B_{s}$ ) trusts other target brokers (represented as $B_{t} \mathrm{~s}$ ). For a specific context $c_{i}, B_{s}$ trusts $B_{t}$ at trust level $T L_{s t}^{c_{i}}$ and this trust level is based on direct experience with $B_{t}$. If $B_{t}$ is unknown to $B_{s}$, $T L_{s t}^{c_{i}}=-1$. The trust levels in the DTT are time stamped to indicate the time of last update and are maintained distributively such that $B_{i}$ maintains row $i$ of the DTT.

Suppose $B_{j}$ is highly trustworthy in the "global" sense, then the DTT should have very high trust levels along column $j$. A DTT is considered consistent if the variation along any given column of the DTT is below a given threshold. Otherwise, the trust model is considered to be inconsistent. With a consistent DTT, when a broker is considered trustworthy by another broker, there is a high probability that other brokers will also find it trustworthy. This property is essential for recommendations to be useful.

Suppose a resource under $B_{s}$ is interested in engaging in a transaction with a resource under $B_{t}$. To determine the suitability of target the resource, $B_{s}$ consults its DTT to obtain the direct trust level and its recommenders in RTT to obtain the reputation. The requests sent to the recommenders can trigger recursive queries yielding a recommender tree. A recommendation tree has DTT lookups at its leaf nodes and RTT lookups at the intermediate nodes. To avoid cycles in the recommender tree, a recommendation request carries the list of visited brokers.

\subsection{Trust Evolution}

After a resource decides to pursue a transaction based on the estimated trust levels, that transaction is either monitored or logged for subsequent analysis by the transaction monitoring proxies (TM proxies) of $B_{s}$ and $B_{t}$. Because a TM proxy is controlled by the broker of the associated domain, TM proxies of $B_{s}$ and $B_{t}$ might evaluate the same transaction differently. The exact definition of "breaches" vary between two TM proxies and some examples include: (a) holding the resources for longer periods that initially requested, (b) trying to access protected local data, (c) instantiating illegal tasks on the resources, and (d) reneging on promises to provide resources [6]. Monitoring the transactions in a real-time fashion can cause significant overhead for trust computation. One way to reduce the overhead is to combine online and offline mechanisms in the monitoring process. [10, 13, 17].

The TTLs obtained by the TM proxies are periodically used to evaluate direct and reputation trust sources. The two sources are evaluated differently. Let $T T L\left(B_{t}, t, c\right)$ denote $B_{t}$ 's TTL for context $c$ at time $t$ and $\operatorname{DTT}\left(B_{s}, B_{t}, t_{s t}, c\right)$ be the current trust level of the DTT entry that corresponds to $B_{s}$ and $B_{t}$ in context $c$ and was last updated on $t_{s t}$. Let $\delta$ be a real number between 0 and 1 . 


$$
\begin{array}{r}
\operatorname{DTT}\left(B_{s}, B_{t}, t, c\right)=(1-\delta) D T T\left(B_{t}, B_{s}, t_{s t}, c\right) \\
+\delta T T L\left(B_{t}, t, c\right)
\end{array}
$$

If $\delta>0.5$, preference is given to the TTL determined through the analysis of the last transaction between the two brokers.

To evaluate the set of recommenders, $B_{s}$ needs to compute the honesty as well as the accuracy measures. A formula similar to above can be used to update the average accuracy. However, the honesty of the recommenders is updated differently. Suppose $H\left(B_{s}, B_{z}\right)$ is the honesty of recommender $B_{z}$ based on the recommendation given to $B_{s}$ and $H_{R T T}\left(B_{s}, B_{z}\right)$ is its honesty as maintained in the RTT based on all previous recommendations. The following simple formula updates the honesty parameter.

$H_{R T T}\left(B_{s}, B_{z}\right)=\min \left(H_{R T T}\left(B_{s}, B_{z}, c\right), H\left(B_{s}, B_{z}\right)\right)$

The above equation penalizes a broker for lying even once. When the honesty value of a broker reaches 0 , it is removed from the recommendation set for a random interval. For the direct trust and accuracy, a weighted moving average algorithm was used to update the parameters. In Section 4.3.1, we show alternative approach and examine their properties.

\section{Performance Evaluation \\ 4.1. Overview}

We conducted a series of simulation studies to examine various properties of the proposed trust model. One performance measure of the trust model is its ability to correctly predict the trust that exists between the brokers. A prediction is considered successful when: (a) a trustworthy broker is predicted as trustworthy and (b) an untrustworthy broker is predicted as untrustworthy. A broker is considered to be trustworthy if its trust level is in $[3,5]$ and considered to be untrustworthy if its trust level is in $[1,2]$. Let the value of the prediction function, $\Phi\left(B_{k}\right)$, be 1 if it correctly predicts $B_{k}$ 's trustworthiness and 0 otherwise. Hence, the success rate (SR) of prediction is computed for $n$ brokers at time $t$ as follows:

$$
S R(t)=\frac{\sum_{k=1}^{n} \Phi\left(B_{k}\right)}{n} \times 100
$$

\subsection{Simulation Model and Setup}

Not all trust brokers are trustworthy at the same level. One of the objectives of the simulation is to model the process of uncovering the trustworthiness of the trust brokers by the trust system through the observation of the transactions that take place among them. We model the trust values that underly among the brokers by an actual direct trust table (ADTT). For simplicity, we assume that these trust relationships do not change for the duration of the simulation time.
The computed direct trust table (CDTT) is another table that is similar to ADTT that is used to keep track of the true trust levels that are revealed by the post mortem processes carried out by the TM proxies. The elicitation of the true trust levels by the post mortem processes are simulated by initially setting CDTT to ADTT plus a random noise generated from $[0,4]$ and setting those values related to the transactions to a small value that is randomly chosen in the range $[0,2]$. This causes the CDTT to contain values closer to "true" values for those relationships for which post mortem analysis have been carried out. In addition to ADTT and CDTT, we maintain a predicted direct trust table (PDTT) to track the evolution of the trust relationships among the brokers. The PDTT values are initially set to -1 and are updated using Equation (2) and the latest values of CDTT.

\subsection{Results and Discussion}

The requests initiating inter-broker transactions are assumed to have a Poisson arrival process. The number of brokers was set to 30 , the size of $R$ was fixed at 4 for all brokers, and the size of $T$ was fixed at 3 for all brokers. The source and the target brokers for each transaction were randomly generated from $[0,29]$. For the simulations performed here, a consistent DTT was used. The length of the monitoring interval is set at $1,5,10$, or 20 meaning that the TM proxy is monitoring every, every fifth, every tenth, or every twentieth transaction, respectively. The value of $\alpha$ is varied from 0 to 1 in 0.5 increments. By varying the monitoring interval, we can examine the sensitivity of the trust model on true trust levels. By varying $\alpha$, we can examine the dependence of the trust model on the different trust components.

\subsubsection{Estimating Trust Levels}

Previously we used an exponential weighted moving average (EWMA) filter for estimating trust levels. One of the drawbacks of this scheme is that it returns high estimates despite periodic occurrences of low values in a sequence of trust values (i.e., a broker can periodically cheat and still maintain a high trust level). The EWMA filter produces an estimate using $O_{t}=\omega O_{t-1}+(1-\omega) O_{c}$, where $O_{t}$ is the newly generated estimate, $O_{t-1}$ is the prior estimate, and $O_{c}$ is the new observation. If $\omega$ is large, the EWMA filter resists rapid changes in individual observations and said to provide stability. For low $\omega$ values, the filter is able to detect changes quickly and said to be agile.

In [12], the stable and agile filters were combined to create a flipflop filter. We develop a variation called modified flipflop, where the agile filter is activated as soon as we detect a drop in value of the trust parameter beyond an acceptable threshold from the previously estimated value. The agile filter quickly downgrades the estimate. For the subsequent estimates, we switch back to the stable filter assuming 
that the trust parameter does not experience any further depreciations.

One of the drawbacks of this filter is that it does not penalize those brokers that continue to periodically cheat. We further modify flipflop to obtain weighted modified flipflop to take periodic cheating into considerations by having a history queue that has the past $n$ values of the trust level. When the $k$ th cheating incident is detected, $k$ low trust values are inserted into the history queue. Because the history queue is limited to $n$ entries, only $n-k$ entries from the past remains in the history queue. In computing the trust level, the history queue entries are weighted such that the weight increases linearly from the head to the tail. From the simulation results shown in Figure 1, we can observe that weighted modified flipflop can detect periodic cheating.

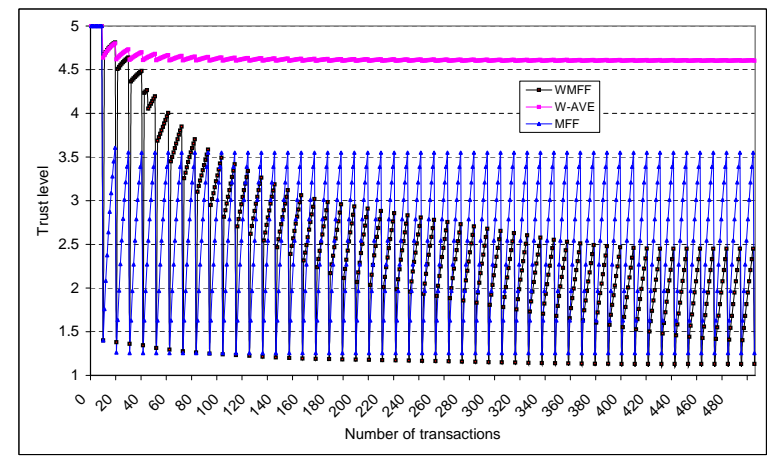

\section{Figure 1. Performance of different filters in detecting malice in updating trust levels.}

\subsubsection{Consistent versus Inconsistent Trust Models}

Here we investigate the dependence of the trust model on the consistence or inconsistence of the DTT. From the simulation results, we found that the success rate is in the range $88.14 \%$ to $100.00 \%$, when the DTT consistent. When the DTT is inconsistent, the success rate is around $50 \%$. This shows that with inconsistent DTT a broker cannot learn actual trust because it is getting conflicting reports on other brokers. An interesting observation is that this low success rate is not affected by a variation in the number of the dishonest brokers.

\subsubsection{Agility of the Trust Model}

Tables 1 and 2 show the success rate of the trust model when using the accuracy alone and accuracy and honesty together, respectively. In Table 1, when there are 0 dishonest brokers, it can be observed that combining direct trust and reputation (i.e., when $\alpha=0.5$ ), outperforms the others (i.e., when $\alpha=1.0$ or when $\alpha=0.0$ ). Because all recommenders are honest, reputation reinforces direct trust and increases the overall success rate. We can also observe that as the trust monitoring interval is increased, the trust model takes longer to reach a given acceptable success rate. In this paper, we arbitrarily set the acceptable success rate as $85 \%$. Tables 1 and 2 show the acceptable successes as darkened entries.

Because the accuracy measure is the difference between a recommender's opinion and the true trust level obtained by the TM proxy, with a shorter monitoring interval, the accuracy will be higher and the trust model will converge faster.

As the dishonest brokers increase to 15 or 20 , any mechanism that relies on the reputation gives poor success rate and the accuracy measure loses its effectiveness. In this case, the solution is to depend on direct trust. However, just using direct trust lowers the convergence rate and also does not exploit the opportunities for cooperation among the brokers, which is a major feature of a network computing system.

To reduce the trust model's sensitivity to dishonest brokers, we use the honesty measure so that dishonest recommenders are filtered out to prevent them from contributing to the recommender network. The overall performance of this strategy is shown in Table 2.

As the number of dishonest brokers increase, we observe the behavior to significantly differ between Tables 1 and 2. In Table 2, combining both components (i.e., direct trust and reputation) gives a higher success rate than relying only on one of them. For example, when the monitoring interval length $=5$, the number of dishonest brokers $=15$, and the number of transactions $=50$, the success rate reaches $77.13 \%$ when $\alpha=1.0$ and $78.62 \%$ when $\alpha=0.0$ but $90.11 \%$ when relying on both. We can conclude that once the dishonest recommenders are filtered out from the recommendation sets, reputation reinforces direct trust and therefore combining these two components yields a higher success rate than relying on only one of them.

The darkened entries in Tables 1 and 2 illustrate the benefit of incorporating honesty into the trust model. The maximum success rates are achieved when the number of dishonest brokers equal zero. As the number of dishonest brokers increase, the number of darkened entries in Table 1 start to reduce. This shows that the accuracy measure is not effective in limiting and preventing the dishonest brokers from influencing the recommenders set. On the other hand, Table 2 show that the number of darkened entries remain almost the same as the number of dishonest brokers increase. This demonstrates the necessity to model honesty and incorporate into the overall trust model.

\section{Case Study: Trust Modeling on Public- Resource Grids}

\subsection{Overview}

In our Grid model, we assume a global resource manager that coordinates the allocation of resources. The global resource manager can be implemented in different ways and 
Table 1. Success rates for a consistent trust model using only the accuracy measure, where $B_{d}$ is number of dishonest brokers and $F_{m}$ the monitoring frequency.

\begin{tabular}{|c|c|c|c|c|}
\hline \multirow{2}{*}{$\begin{array}{l}B_{d} / \\
F_{m}\end{array}$} & \multirow{2}{*}{$\begin{array}{c}\alpha \\
\text { value }\end{array}$} & \multicolumn{3}{|c|}{ Number of transactions per relation } \\
\hline & & 5 & 50 & 150 \\
\hline \multirow[t]{3}{*}{$0 / 1$} & 1.0 & $71.83 \%$ & $99.86 \%$ & $100.00 \%$ \\
\hline & 0.5 & $86.55 \%$ & $99.54 \%$ & $99.54 \%$ \\
\hline & 0.0 & $79.77 \%$ & $95.29 \%$ & $99.01 \%$ \\
\hline \multirow[t]{3}{*}{$\overline{0 / 5}$} & 1.0 & $57.01 \%$ & $86.90 \%$ & 99.31\% \\
\hline & 0.5 & $60.57 \%$ & $97.47 \%$ & $99.11 \%$ \\
\hline & 0.0 & $60.23 \%$ & $86.32 \%$ & $89.77 \%$ \\
\hline \multirow[t]{3}{*}{$0 / 10$} & 1.0 & $54.02 \%$ & $77.36 \%$ & $92.18 \%$ \\
\hline & 0.5 & $56.44 \%$ & $88.16 \%$ & 98.85\% \\
\hline & 0.0 & $54.94 \%$ & $79.66 \%$ & $88.74 \%$ \\
\hline \multirow[t]{3}{*}{$0 / 20$} & 1.0 & $51.95 \%$ & $64.37 \%$ & $81.84 \%$ \\
\hline & 0.5 & $53.68 \%$ & $75.86 \%$ & 95.98\% \\
\hline & 0.0 & $51.84 \%$ & $69.54 \%$ & $82.53 \%$ \\
\hline \multirow[t]{3}{*}{ 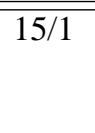 } & 1.0 & $71.84 \%$ & $99.89 \%$ & $100.00 \%$ \\
\hline & 0.5 & $76.90 \%$ & $91.95 \%$ & $91.49 \%$ \\
\hline & 0.0 & $55.86 \%$ & $62.99 \%$ & $64.25 \%$ \\
\hline \multirow[t]{3}{*}{$15 / 5$} & 1.0 & $57.01 \%$ & $86.90 \%$ & $99.31 \%$ \\
\hline & 0.5 & $55.17 \%$ & $85.52 \%$ & $89.77 \%$ \\
\hline & 0.0 & $50.69 \%$ & $53.91 \%$ & $54.60 \%$ \\
\hline \multirow[t]{3}{*}{$15 / 10$} & 1.0 & $54.02 \%$ & $77.36 \%$ & 92.18\% \\
\hline & 0.5 & $52.99 \%$ & $75.63 \%$ & $88.28 \%$ \\
\hline & 0.0 & $49.77 \%$ & $51.72 \%$ & $52.41 \%$ \\
\hline \multirow[t]{3}{*}{$15 / 20$} & 1.0 & $51.95 \%$ & $64.37 \%$ & $81.84 \%$ \\
\hline & 0.5 & $51.03 \%$ & $63.44 \%$ & $81.61 \%$ \\
\hline & 0.0 & $50.92 \%$ & $50.11 \%$ & $52.18 \%$ \\
\hline \multirow[t]{3}{*}{$20 / 1$} & 1.0 & $71.67 \%$ & $99.89 \%$ & $100.00 \%$ \\
\hline & 0.5 & $72.01 \%$ & $89.54 \%$ & $89.54 \%$ \\
\hline & 0.0 & $49.89 \%$ & $57.13 \%$ & $57.24 \%$ \\
\hline \multirow[t]{3}{*}{$20 / 5$} & $\overline{1.0}$ & $57.09 \%$ & $\overline{86.90 \%}$ & $\overline{99.31 \%}$ \\
\hline & 0.5 & $54.60 \%$ & $76.90 \%$ & $83.91 \%$ \\
\hline & 0.0 & $44.14 \%$ & $45.75 \%$ & $47.47 \%$ \\
\hline \multirow[t]{3}{*}{$\overline{20 / 10}$} & 1.0 & $54.02 \%$ & $77.36 \%$ & $92.18 \%$ \\
\hline & 0.5 & $52.07 \%$ & $68.28 \%$ & $79.20 \%$ \\
\hline & 0.0 & $46.32 \%$ & $44.60 \%$ & $45.75 \%$ \\
\hline \multirow[t]{3}{*}{$20 / 20$} & 1.0 & $51.95 \%$ & $64.37 \%$ & $81.84 \%$ \\
\hline & 0.5 & $51.90 \%$ & $62.87 \%$ & $73.68 \%$ \\
\hline & 0.0 & $49.08 \%$ & $46.09 \%$ & $44.25 \%$ \\
\hline
\end{tabular}

Table 2. Success rates for a consistent trust model using the accuracy and the honesty measures, where $B_{d}$ is number of dishonest brokers and $F_{m}$ the monitoring frequency.

\begin{tabular}{|c|c|c|c|c|}
\hline \multirow{2}{*}{$\begin{array}{l}B_{d} / \\
F_{m}\end{array}$} & \multirow{2}{*}{$\begin{array}{c}\alpha \\
\text { value }\end{array}$} & \multicolumn{3}{|c|}{ Number of transactions per relation } \\
\hline & & 5 & 50 & 150 \\
\hline \multirow[t]{3}{*}{$0 / 1$} & 1.0 & $71.22 \%$ & $100.00 \%$ & $100.00 \%$ \\
\hline & 0.5 & $87.36 \%$ & $\mathbf{9 9 . 5 4 \%}$ & $100.00 \%$ \\
\hline & 0.0 & $80.15 \%$ & $95.67 \%$ & $100.00 \%$ \\
\hline \multirow[t]{3}{*}{$0 / 5$} & 1.0 & $56.78 \%$ & $88.16 \%$ & $100.00 \%$ \\
\hline & 0.5 & $62.18 \%$ & $98.16 \%$ & $100.00 \%$ \\
\hline & 0.0 & $60.46 \%$ & $87.47 \%$ & $90.03 \%$ \\
\hline \multirow[t]{3}{*}{$0 / 10$} & 1.0 & $54.28 \%$ & $77.13 \%$ & 92.10\% \\
\hline & 0.5 & $56.67 \%$ & $90.34 \%$ & $99.01 \%$ \\
\hline & 0.0 & $55.63 \%$ & $80.23 \%$ & 88.14\% \\
\hline \multirow[t]{3}{*}{$0 / 20$} & 1.0 & $51.49 \%$ & $66.44 \%$ & $82.79 \%$ \\
\hline & 0.5 & $52.18 \%$ & $76.32 \%$ & $95.66 \%$ \\
\hline & 0.0 & $51.84 \%$ & $70.57 \%$ & $82.70 \%$ \\
\hline \multirow[t]{3}{*}{$15 / 1$} & 1.0 & $71.20 \%$ & $100.00 \%$ & $100.00 \%$ \\
\hline & 0.5 & $87.70 \%$ & $99.67 \%$ & $100.00 \%$ \\
\hline & 0.0 & $77.82 \%$ & $97.01 \%$ & $100.00 \%$ \\
\hline \multirow[t]{3}{*}{$15 / 5$} & 1.0 & $56.78 \%$ & $\mathbf{8 8 . 1 6 \%}$ & $100.00 \%$ \\
\hline & 0.5 & $63.45 \%$ & $\mathbf{9 7 . 8 2 \%}$ & $100.00 \%$ \\
\hline & 0.0 & $62.41 \%$ & $86.44 \%$ & $91.78 \%$ \\
\hline \multirow[t]{3}{*}{$15 / 10$} & 1.0 & $54.25 \%$ & $77.13 \%$ & $92.19 \%$ \\
\hline & 0.5 & $55.40 \%$ & $90.11 \%$ & $100.00 \%$ \\
\hline & 0.0 & $55.98 \%$ & $78.62 \%$ & $\mathbf{8 8 . 9 1 \%}$ \\
\hline \multirow[t]{3}{*}{$15 / 20$} & 1.0 & $51.49 \%$ & $66.44 \%$ & $82.02 \%$ \\
\hline & 0.5 & $53.33 \%$ & $77.47 \%$ & $96.59 \%$ \\
\hline & 0.0 & $53.79 \%$ & $68.97 \%$ & $84.14 \%$ \\
\hline \multirow[t]{3}{*}{$20 / 1$} & 1.0 & $71.12 \%$ & $100.00 \%$ & $100.00 \%$ \\
\hline & 0.5 & $89.08 \%$ & $99.89 \%$ & $100.00 \%$ \\
\hline & 0.0 & $82.18 \%$ & $\mathbf{9 8 . 8 5 \%}$ & $100.00 \%$ \\
\hline \multirow[t]{3}{*}{$20 / 5$} & 1.0 & $56.78 \%$ & $88.16 \%$ & $100.00 \%$ \\
\hline & 0.5 & $63.22 \%$ & $98.16 \%$ & $100.00 \%$ \\
\hline & 0.0 & $61.72 \%$ & $\mathbf{8 9 . 8 9 \%}$ & $94.01 \%$ \\
\hline \multirow[t]{3}{*}{$20 / 10$} & $\overline{1.0}$ & $54.25 \%$ & $\overline{77.10 \%}$ & $\overline{92.13 \%}$ \\
\hline & 0.5 & $55.98 \%$ & $93.33 \%$ & $100.00 \%$ \\
\hline & 0.0 & $55.98 \%$ & $80.52 \%$ & $89.47 \%$ \\
\hline \multirow[t]{3}{*}{$20 / 20$} & 1.0 & $51.49 \%$ & $66.86 \%$ & $82.49 \%$ \\
\hline & 0.5 & $52.53 \%$ & $78.31 \%$ & $94.93 \%$ \\
\hline & 0.0 & $52.30 \%$ & $70.57 \%$ & $85.01 \%$ \\
\hline
\end{tabular}


the simplest among them is the centralized approach. Alternative approaches include hierarchical and federated. Irrespective of the approach, the resource manager maintains information regarding the resources in either aggregated or raw form and makes the allocation decisions using this information.

In this section, we enhance the global resource manager of the Grid model such that it tracks the trust relationships among the different resource domains and brings together only those domains that have high levels of trust among them in any given allocation. As a result, the resource forming a virtual collection have higher trust among them and can provide higher levels of assurance on the delivered performance.

\subsection{A Trust Model for Public-Resource Grids}

In our Grid model, a public-resource Grid is composed of several domains, where each domain is managed by a broker. For trust-aware resource management purposes, we can consider a resource presented by the broker to have the following attributes: (a) type of contexts (ToCs) it supports and (b) a trust level for each ToC. Because a broker is responsible for representing the resources in the trust model, the broker and the resource negotiate on the ToCs and the corresponding trust levels. Associating a trust level with each ToC provides the flexibility of selectively opening services to clients. Similarly, client domains have their own trust attributes including: (a) ToCs sought and (b) trust levels associated with the ToCs.

The DTT provides $T L_{i j}^{c_{k}}$, which is the offered trust level (OTL) that $B_{i}$ offers to $B_{j}$ to engage in activity within context $c_{k}$. Suppose we have client $X$ from $B_{i}$ wanting to engage in activities within contexts $c_{p}, c_{q}$, and $c_{r}$ on resource $Y$ in $B_{j}$. Because resource and client domains inherit the trust levels from the broker they are associated with, we can compute the OTL for the composite activity between $X$ and $Y$, i.e., OTL $=\min \left(T L\right.$ for $c_{p}, T L$ for $c_{q}, T L$ for $\left.c_{r}\right)$. There are two required trust levels (RTLs), one from the client side and the other from the resource side. If the OTL is greater than or equal to the maximum of client and resource RTLs, then the activity can proceed with no additional overhead. Otherwise, there will be a risk involved in carrying out the activity. The expected risk factor (ERF) for given RTL and OTL values are provided by RTL - OTL and is 0 , when RTL - OTL $<0$.

\subsection{A Model for Trust Aware Resource Manage- ment}

The resource management model presented here is based on the following assumptions: (a) scheduler is organized centrally, (b) tasks are mapped non-preemptively, (c) tasks are indivisible (i.e., a task cannot be distributed over multiple machines), and (d) tasks are independent (i.e., there is no data dependency among the different tasks).
For request $r_{j}$, let $t\left(r_{j}\right)$ and $c\left(r_{j}\right)$ denote the task and originating client, respectively. Suppose that we have a set of tasks $t\left(r_{0}\right) \ldots t\left(r_{n-1}\right)$ and a set of machines $m_{0} \ldots m_{k-1}$. We can allocate the tasks onto the machines in $n \times k$ different ways. With each allocation, we can associate a completion time $\left(\alpha\left(t\left(r_{j}\right), m_{i}\right)\right)$ and an ERF $\left(\beta\left(t\left(r_{j}\right), m_{i}\right)\right)$. The completion time indicates when $m_{i}$ is going to be available after completing task $t\left(r_{j}\right)$, whereas $\beta\left(t\left(r_{j}\right), m_{i}\right)$ indicates the expected risk, when assigning $t\left(r_{j}\right)$ to $m_{i}$. Further, let $\gamma_{i}$ be the available time of machine $m_{i}$ after executing all prior requests assigned to it.

\subsection{Resource Management Algorithms}

In this section, we show how a simple resource management heuristic can be modified to perform trust aware resource management. The objective of this exercise is to show the utility of the trust model and not to solve the resource management problem optimally. In this study, we choose the min-min heuristic [14] as the base algorithm.

The min-min is a trust unaware algorithm and it works as follows. Let $\operatorname{EEC}\left(t\left(r_{j}\right), m_{i}\right)$ be the expected execution cost of $t\left(r_{j}\right)$ on machine $m_{i}$. In addition, let ECC $\left(t\left(r_{j}\right), m_{i}\right)$ denote the expected completion cost of $t\left(r_{j}\right)$ on machine $m_{i}$, which is computed by adding the EEC of $t\left(r_{j}\right)$ on machine $m_{i}$ and the available time of $m_{i}$. The min-min heuristic has two phases. In the first phase: For each task $t\left(r_{j}\right)$, the machine that gives the earliest expected completion cost is determined using the EEC matrix and the machine available times. In the second phase, task $t\left(r_{k}\right)$ with the minimum earliest completion cost is found and is assigned to the corresponding machine. The goal of the min-min heuristic is to assign a set of requests $\left\{r_{0} \ldots r_{n-1}\right\}$ such that $\left\{\max _{i}\left\{\gamma_{i}\right\}\right\}$ is minimized for $0 \leq i<m$, where $n$ is the number of requests and $m$ is the number of machines.

\subsubsection{Trade-off Algorithm}

With trust aware resource management, we need to perform allocation such that the overall completion times are minimized while the risk associated with the allocations are simultaneously reduced. This "biobjective" allocation is harder to achieve. This algorithm trades off one objective for another by weighing them differently.

The trade-off algorithm works as follows. For each $t\left(r_{j}\right)$, it finds the maximum $\alpha\left(t\left(r_{j}\right), m_{i}\right)$ and the maximum $\beta\left(t\left(r_{j}\right), m_{i}\right)$ and normalizes the entries in matrices ECC and ERF by these two numbers, respectively. This results in normalized ECC and ERF matrices. Because the completion time (i.e., ECC matrix) changes with each assignment, the ECC matrix should be recomputed and renormalized with every assignment.

Let $w_{c}$ and $w_{r}$ represent the weights associated with the completion time and risk components, respectively. The trade-off process can be carried out by changing these weights. It should be noted that the relative value of these 
weights do not imply that some risk value is equivalent to some value of completion time. When the trade-off is applied, we are dealing with normalized completion times and risks. Once the biobjective trust-aware resource management problem is transformed into a uniobjective formulation through this trade-off process, a single parameter minimization heuristic such as min-min can be directly applied. This is exactly how the problem is solved in this section.

\subsubsection{Maximum Risk Algorithm}

Suppose $R_{\max }$ is the maximum risk that the resource manager is willing to take for any individual allocation. A penalty factor $\operatorname{diff}\left(\beta\left(\mathrm{t}\left(\mathrm{r}_{\mathrm{j}}\right), \mathrm{m}_{\mathrm{i}}\right)-\mathrm{R}_{\max }\right) \mathrm{Q}$ is added to $\alpha\left(t\left(r_{j}\right), m_{i}\right)$, where $Q$ is a large penalty factor and $\operatorname{diff}(\mathrm{x})=0$ if $x<0$ and 1 otherwise. Once the penalty factor is added to the completion times, the min-min algorithm is used to select the task-to-machine allocations. By adding the penalty factor, we are able to avoid high risk allocations.

\subsection{Performance of Trust Aware Resource Man- agement}

To highlight the benefits of trust aware resource management, we investigate two factors that impact performance: (a) makespan [14] for the complete schedule (b) makespan variability. The makespan is defined as $\max _{\mathrm{i} \in \mathrm{K}}\left(\alpha\left(\mathrm{t}\left(\mathrm{r}_{\mathrm{j}}\right), \mathrm{m}_{\mathrm{i}}\right)\right)$ and is a measure of the throughput of the whole resource allocation process. The makespan variability is the defined as the variation in makespan as the risk penalty value $Q$ changes.

To simulate the impact of risk, we compute the expected completion time of a request $r_{j}$ mapped onto machine $m_{i}$ without considering the risk penalty and then compute the actual completion time for the same mapping by adding risk penalty to the above completion time. The risk penalty determines the cost due to misbehaving resources. The ERF (i.e., $\left.\beta\left(t\left(r_{j}\right), m_{i}\right)\right)$ indicates how likely it is to incur the risk penalty. For example, ERF of 3 indicates that there is a $(3 / 5) 100=20 \%$ chance of incurring the risk penalty. The actual risk penalty itself is computed as a fraction $x$ of the corresponding EEC value.

The resource allocation process was simulated using a discrete event simulator with Poisson request arrivals. We consider 20 resource domains. The source and target domains were randomly picked from $[1,20]$. The ToCs required for each request were randomly generated from [1, 4] meaning that each $t\left(r_{i}\right)$ involves at least one ToC but no more than four ToCs. The two RTLs were randomly generated from $[1,6]$ and the OTLs were randomly generated from [1,5]. The simulations results for 10,000 tasks and 20 machines are shown in Table 3.

As expected, the min-min algorithm that only considers completion times perform best in terms of the expected makespan. However, the actual makespan varies signifi- cantly from the expected makespan due to the addition of the risk penalty. The mapping computed by min-min gives the highest variability on the makespan (i.e., considering risk while mapping results in mappings that are resistant to risk related variations at run time. This "robustness" of the resource allocation is highly desirable because if the expected makespan of an allocation is not representative of the actual makespan, then the resource manager cannot do meaningful capacity planning.

Table 3. Comparison of makespan and robustness of various resource management algorithms using a consistent trust model with $w_{r}=1-w_{c}$.

\begin{tabular}{|c|c|c|c|c|}
\hline $\begin{array}{c}x \\
\text { value }\end{array}$ & $\begin{array}{l}\text { RMS } \\
\text { algo. }\end{array}$ & $\begin{array}{c}w c \\
\text { value }\end{array}$ & $\begin{array}{l}\text { Expected } \\
\text { makespan }\end{array}$ & $\begin{array}{c}\text { Actual } \\
\text { makespan }\end{array}$ \\
\hline \multirow[t]{8}{*}{0.01} & min-min & NA & $16,011.28$ & $16,094.54$ \\
\hline & \multirow[t]{6}{*}{ trade-off } & 0.0 & $178,672.41$ & $178,686.17$ \\
\hline & & 0.2 & $40,323.55$ & $40,325.78$ \\
\hline & & 0.4 & $39,474.82$ & $39,477.04$ \\
\hline & & 0.6 & $40,244.76$ & $40,244.76$ \\
\hline & & 0.8 & $35,273.99$ & $35,274.98$ \\
\hline & & 1.0 & $15,985.72$ & $16,055.42$ \\
\hline & max. risk & NA & $30,195.42$ & $30,211.99$ \\
\hline \multirow[t]{8}{*}{1.0} & min-min & NA & $\overline{16,011.28}$ & $24,336.83$ \\
\hline & \multirow[t]{6}{*}{ trade-off } & 0.0 & $178,672.41$ & $180,048.14$ \\
\hline & & 0.2 & $40,323.55$ & $40,546.34$ \\
\hline & & 0.4 & $39,474.82$ & $39,697.60$ \\
\hline & & 0.6 & $40,244.76$ & $40,244.76$ \\
\hline & & 0.8 & $35,273.99$ & $35,372.55$ \\
\hline & & 1.0 & $15,985.72$ & $25,297.04$ \\
\hline & max. risk & $\overline{\mathrm{NA}}$ & $30,195.42$ & $31,853.08$ \\
\hline \multirow[t]{8}{*}{10.0} & $\overline{\text { min-min }}$ & $\overline{\mathrm{NA}}$ & $16,011.28$ & $\overline{101,871.41}$ \\
\hline & \multirow[t]{6}{*}{ trade-off } & 0.0 & $178,672.41$ & 192.429 .71 \\
\hline & & 0.2 & $40,323.55$ & $42,899.47$ \\
\hline & & 0.4 & $39,474.82$ & $42,451.45$ \\
\hline & & 0.6 & $40,244.76$ & $42,798.63$ \\
\hline & & 0.8 & $35,273.99$ & $41,317.49$ \\
\hline & & 1.0 & $15,985.72$ & $110,686.16$ \\
\hline & max. risk & $\mathrm{NA}$ & $30,195.42$ & $49,040.93$ \\
\hline
\end{tabular}

\section{Related Work}

A model for supporting behavior trust based on experience and reputation is proposed in [1]. This trust-based model allows entities to decide which other entities are trustworthy and also allows entities to tune their understanding of another entity's recommendations. Each entity keeps two sets: (a) set $Q$ for entities directly trusted and (b) set $R$ for recommenders. One of the drawbacks of this model 
is its use on an exponentially weighted moving average algorithm for updating $Q$ and $R$. In this approach, a recommender can give intentionally damaging recommendations about few domains and maintain high overall accuracy. Because our model uses the honesty concept, such recommenders will be detected and isolated from $R$. Further, the scalability of the model is not explicitly addressed in this study. In our model, the scalability issue is addressed by the aggregation scheme.

A reputation-based approach for extending Gnutella-like environments is proposed in [5], where an entity uses a polling protocol to query and select target entities Each entity maintains information on its own experience with target entities and shares such experiences when polled by other entities. This approach has no mechanism to filter out dishonest entities from the reputation network. An entity broadcasts its request to all of its neighbors regardless of their honesty. This practice is not just inefficient, but also gives continued opportunities for dishonest entities to damage and influence the reputation network. Because this approach uses a voting scheme to determine the truth, an entity can be fooled if the majority of recommenders are dishonest. Further, this model is based only on reputation and deals with file sharing or file exchange.

A trust management in a $\mathrm{P} 2 \mathrm{P}$ information system is proposed in [2], where the focus is on implementing a generic scalable infrastructure to deploy any trust model. A simple trust model was proposed, where entities file complaints based on bad experiences they had while interacting with other entities. One limitation of this model is that it is based on a binary trust scale (i.e., an entity is either trustworthy or not). Hence, once there is a complaint filed against entity $p$, $p$ is considered untrustworthy even though it has been trustworthy for all previous transactions. Also, this approach has no mechanism for preventing a malicious entity from inserting arbitrary number of complaints and potentially causing a denial of service attack.

A trust model for P2P content distribution networks is presented in [15], where web servers can cooperate to replicate their documents worldwide. This model is based on recommendations and again uses an EWMA algorithm, when updating the recommender set. Hence, dishonest entities can cheat every $n$ transactions and still be considered trustworthy. A decentralized trust model Poblano [4] is implemented in a P2P fashion for the Project JXTA [9]. This model is based on recommendations and provides algorithms to determine the trustworthiness of a peer's data based on its reputation. This approach is used to perform reputation guided searching or to securely distribute signed certificates among peers.

Assuming that less than $50 \%$ of a population of entities are malicious, a simple reputation polling mechanism is presented in [16]. In this recommendation scheme, an entity's trustworthiness is determined through majority voting.

A reputation management model for a multiagent system is proposed in [19]. An entity determines the trustworthiness of a correspondent by combining its local experience with the testimonies of recommenders regarding the same correspondent. Again, this approach does not prevent dishonest entities from generating spurious ratings and assumes that the majority of entities offer honest ratings to cancel the effect of dishonest entities.

The trust brokering system presented in this paper also differs from the mechanisms we presented in a previous paper [3]. This paper introduces the aggregation scheme where a broker represents a collection of resources. This improves the scalability of the mechanism both in terms of the number of resources that can be handled by the system and the number of transactions required to converge at the appropriate trust values. Further, this paper introduces the notion of honesty to prevent dishonest recommenders from polluting the recommendation network and a new framework for incorporating trust into resource management systems. The framework presented here relaxes some of the assumptions made in [3].

In summary, one of the major differences between our trust model and the ones examined above is the separation of honesty and trustworthiness in our model. In addition, to the best of our knowledge, no existing literature directly addresses the problem of integrating trust into resource management schemes.

\section{Conclusions and Future Work}

In this paper, we presented a trust model for publicresource Grid systems. The public-resource Grid systems enable resources without a priori trust relationships to participate as providers in a Grid system. In such conditions, it becomes essential for the resource manager to be trust cognizant to avoid bringing together untrusting parties under a single virtual cluster to solve a given problem.

In this paper, we present a trust brokering system that can be used in a public-resource Grid system. Extensive simulation studies were conducted to evaluate the model under various conditions.

Our trust model uses an accuracy concept to enable peer review-based mechanisms to function with imprecise trust metrics, the imprecision is introduced by peers evaluating the same situation differently. Simulation results show that the reputation-based trust model reaches an acceptable level of capability after a certain number of transactions. However, as the number of dishonest domains increase, the model becomes slow in reaching the acceptable level of capability.

To reduce the trust model's sensitivity to dishonest domains, we introduced an honesty concept to handle the sit- 
uation where domains intentionally lie about other domains for their own benefit. Simulation results indicate that incorporating the honesty concept into the trust model, limits the effect of dishonest domains by preventing them from providing recommendations.

Another feature of our model is the flexibility to weigh direct trust and reputation differently. Simulation results show that it is better to rely on direct trust when honesty is not used. This can be explained by observing that due to malicious recommenders the reputation is tainted and using it can only lead to incorrect decisions. When the honesty is used to isolate the malicious recommenders, we assured of an honest of set of recommenders. In this situation, simulation results indicate that significant benefits can be obtained by using reputations.

Another significant advantage of our scheme is that our scheme does not depend on a majority opinion as previous schemes did. Therefore, our scheme can work even when majority of the recommenders are malicious. Actually as the malicious number of recommenders increase, the recommenders providing recommendations to a query reduces. The number of recommenders also provides another measure of trust on the overall system because all the recommenders are considered honest.

As an application of our trust model, we incorporate trust-awareness into the resource management system such that the allocation decisions are trust cognizant. The simulations performed to evaluate the effectiveness of the modifications indicate that due to trust awareness, the overall performance of the resource management system improves in different ways.

In summary, our trust model provides two levels of incentives for domains. First, by modeling honesty, the trust model gives incentive to recommenders to truthfully give recommendations and cooperate. If a recommender is dishonest, it will be isolated from the rest of the environment. Second, by modeling trust, the model provides incentives for the domains to be trustworthy and behave as expected. Trust-aware resource management system is a concrete example, where trust levels maintained by the trust model are used in determining the privileges of a domain.

\section{Acknowledgment}

This research is supported by a TRLabs Scholarship, University of Manitoba Graduate Fellowship, and by Natural Sciences and Engineering Research Council of Canada Discovery Grant RGP220278. The equipments used were supported by a Canada Foundation for Innovation Grant.

\section{References}

[1] A. Abdul-Rahman and S. Hailes. Supporting trust in virtual communities. In Hawaii Int'l Conf. System Sciences, Jan. 2000 .
[2] K. Aberer and Z. Despotovic. Managing trust in a peer-2peer information system. In 10th Int'l Conf. Information and Knowledge Management (CIKM'01), pages 310-317, Nov. 2001.

[3] F. Azzedin and M. Maheswaran. Integrating trust into Grid resource management systems. In 2002 Int'l Conf. Parallel Processing (ICPP '02), pages 47-54, Aug. 2002.

[4] R. Chen and W. Yeager. Poblano: A distributed trust model for peer-to-peer networks. http://security.jxta.org, 2001.

[5] E. Damiani, S. D. C. di Vimercati, S. Paraboschi, P. Samarati, and F. Violante. A reputation-based approach for choosing reliable resources in peer-to-peer networks. In 9th ACM Conf. Computer and Communications Security, pages 207216, Nov. 2002.

[6] R. Dingledine, M. J. Freedman, and D. Molnar. Accountability. In A. Oram, editor, Peer-to-Peer: Harnessing the Power of Disruptive Technologies, pages 271-340. O'Reilly and Associates, Sebastopol, CA, 2001.

[7] I. Foster and A. Iamnitchi. On death, taxes, and the convergence of peer-to-peer and Grid computing. In 2nd Int'l. Workshop on Peer-to-Peer Systems (IPTPSO3), Feb. 2003.

[8] I. Foster, C. Kesselman, and S. Tuecke. The anatomy of the Grid: Enabling scalable virtual organizations. Int'l J. Supercomputer Applications, 2001.

[9] L. Gong. JXTA: A network programming environment. IEEE Internet Computing, 5(3):88-95, May/June 2001.

[10] N. Habra, B. L. Chalier, A. Mounji, and I. Mathieu. ASAX: Software architecture and rule-based language for universal audit trail analysis. In European Symposium on Research in Computer Security (ESORIC'92), pages 435-450, Nov. 1992.

[11] I. Foster and C. Kesselman (eds.). The Grid: Blueprint for a New Computing Infrastructure. Morgan Kaufmann, San Fransisco, CA, 1999.

[12] M. Kim and B. Noble. Mobile networks estimation. In 7th Annual Conf. Mobile Computing and Networking, July 2001.

[13] T. F. Lunt. Detecting intruders in computer systems. In Conf. Auditing and Computer Technology, 1993.

[14] M. Maheswaran, S. Ali, H. J. Siegel, D. Hensgen, and R. F. Freund. Dynamic mapping of a class of independent tasks onto heterogeneous computing systems. J. Parallel and Distributed Computing, 59(2):107-131, Nov. 1999.

[15] G. Pierre and M. van Steen. A trust model for peer-to-peer content distribution networks. Nov. 2001.

[16] S. Sen and N. Sajja. Robustness of reputation-based trust: Boolean case. In 1st Int'l Joint Conf. Autonomous Agents and Multi-Agent Systems (AAMAS-02), pages 288-293, July 2002.

[17] S. E. Smaha and J. Winslow. Misuse detection tools. J. Computer Security, 10(1):39-49, Jan. 1994.

[18] M. Waldman, L. F. Cranor, and A. Rubin. Trust. In A. Oram, editor, Peer-to-Peer: Harnessing the Power of Disruptive Technologies, pages 271-340. O'Reilly and Associates, Sebastopol, CA, 2001.

[19] B. Yu and M. P. Singh. An evidential model for distributed reputation management. In 1st Int'l Joint Conf. Autonomous Agents and Multi-Agent Systems (AAMAS-02), pages 294301, July 2002. 